\title{
THE POLYNOMIAL OF A DIRECTED GRAPH
}

\author{
A. J. HOFFMAN ${ }^{1}$ AND M. H. MCANDREW
}

1. Introduction. In a recent paper [1], the concept of the polynomial of an undirected graph was introduced, and it was pointed out that (i) a graph has a polynomial if and only if it is regular and connected, and (ii) various previous studies (see the references in [1]) were special cases of the problem: find all graphs having the same polynomial.

In this paper, we prove the analogue of (i) for directed graphs, and, in addition, obtain some results of type (ii) for a class of directed graphs arising from a mesh on a torus.

2. On the existence of polynomials. Let $G$ be a directed graph on $n$ vertices, with at most one edge from vertex $i$ to vertex $j$, and no edge from $i$ to $i$. For each vertex $i$, let $d_{i}$ be the number of edges with terminal vertex $i, e_{i}$ be the number of edges with initial vertex $i$. $G$ is said to be strongly regular if $d_{i}=e_{i}=d, i=1, \cdots, n ; G$ is said to be strongly connected if, for any vertices $i$ and $j, i \neq j$, there is a directed path from $i$ to $j$.

Let $A(G)=A$ be the adjacency matrix of $G$, i.e.,

$$
a_{i j}= \begin{cases}1 & \text { if there is an edge from } i \text { to } j, \\ 0 & \text { otherwise. }\end{cases}
$$

Let $u$ be the vector of order $n$ every entry of which is unity, $J$ the matrix of order $n$ every column of which is $u$.

Theorem 1. (i) There exists a polynomial $P(x)$ such that

$$
J=P(A)
$$

if and only if $G$ is strongly connected and strongly regular.

(ii) The unique polynomial of least degree satisfying (2.1) is $n S(x) / S(d)$ where $(x-d) S(x)$ is the minimal polynomial of $A$ and $d$ is the valence of $G$.

(iii) If $P(x)$ is that polynomial of least degree satisfying (2.1), then the valence of $G$ is the greatest real root of $P(x)=n$.

Proof. Assume (2.1). Let $i, j$ be distinct vertices of $G$. By (2.1), there is some integer $k$ such that $A^{k}$ has a positive entry in position

Received by the editors October 1, 1963.

1 This research was supported in part by the Office of Naval Research, under Contract No. Nonr. 3775(00), NR 047040. 
$(i, j)$, i.e., there is some $k$-step path from $i$ to $j$. So $G$ is strongly connected. Further, from (2.1), $J$ commutes with $A$. But the $(i, j)$ th entry of $A J$ is $e_{i}$, and the $(i, j)$ th entry of $J A$ is $d_{j}$. Thus $e_{i}=d_{j}$ for all $i$ and $j$, so $G$ is strongly regular.

To prove the converse of (i), assume $G$ strongly connected and strongly regular. From the strong regularity, $u$ is a left and right eigenvector of $A$, corresponding to the eigenvalue $d$. Hence, if $d$ has multiplicity greater than 1 , it must have at least one more eigenvector associated with it. But from the strong connectedness, using a standard argument [1], $u$ is the only eigenvector corresponding to $d$. It follows that, if $R(x)$ is the minimal polynomial of $A$, and if $S(x)=R(x) /(x-d)$ then $S(d) \neq 0$. We then have

$$
0=R(A)=(A-d I) S(A) \text {. }
$$

Since $R(A) v=0$ for all vectors $v$, it follows from (2.2) that

$$
(A-d I) S(A) v=0
$$

so $S(A) v=\alpha u$ for some $\alpha$.

If $(v, u)=0$ then $\left(A^{k} v, u\right)=\left(v,\left(A^{T}\right)^{k} u\right)=d^{k}(v, u)=0$ for every $k$ and so $(S(A) v, u)=0$. Therefore, $0=(S(A) v, u)=(\alpha u, u)=n \alpha$, i.e., $\alpha=0$.

Thus $S(A) v=0$ for all $v$ such that $(v, u)=0$; further, $S(A) u=S(d) u$. Hence $n S(A) / S(d)=J$, i.e. a polynomial which will accomplish (2.1) is

$$
P(x)=\frac{n}{S(d)} S(x)
$$

This completes the proof of (i); (ii) follows since (2.3) has smaller degree than the minimal polynomial of $A$.

To prove (iii) we note that $A$ is non-negative and has row and column sums $d$. Thus, by [2], the eigenvalues of $A$ are all of absolute value $\leqq d$. The roots of $P(x)$ are eigenvalues of $A$ and hence for real $x>d,|P(x)|$ is a monotone increasing function of $x$. From (2.3), $P(d)=n$ and so, since $P(x)$ is a real polynomial, $P(x)>n$ for $x>d$.

This completes the proof ${ }_{p}$ of the theorem. We call (2.3) the polynomial belonging to $G$ (and also say that $G$ belongs to the polynomial).

3. A graph on a torus. For any positive integer $t$ let $G_{t}$ be the graph whose vertices are all ordered pairs $(i, j)$ of residues $\bmod t$ and whose edges go from $(i, j)$ to $(i, j+1)$ and $(i+1, j)$ for all $i, j$. Clearly $G_{t}$ is strongly regular of valence 2 , and strongly connected. We now derive its polynomial. 
Let $\lambda, \mu$ be arbitrary (not necessarily distinct) $t$ th roots of unity. Let $v$ be the vector whose $(i, j)$ th component is $\lambda^{i} \mu^{i}$. If $A$ is the adjacency matrix of $G_{t}$ then $A v=(\lambda+\mu) v$. Further, different vectors $v_{1}, v_{2}$ have as their scalar product $\sum_{i, j} \lambda_{1}^{i} \mu_{1}^{j} \lambda_{2}^{-i} \mu_{2}^{-i}$, which is zero unless $\lambda_{1}=\lambda_{2}$ and $\mu_{1}=\mu_{2}$. Hence the set of vectors corresponding to the $t^{2}$ choices of the pair $\lambda, \mu$ form a complete orthogonal set of right eigenvectors. From this it follows that $A$ is normal and that the minimal polynomial of $A$ has no repeated factors. Hence, by Theorem 1 , the polynomial belonging to $G_{t}$ is

$$
P_{t}(x)=\frac{t^{2}}{S(d)} S_{t}(x)
$$

where

$$
S_{t}(x)=\frac{\Pi(x-\rho)}{x-2},
$$

the product being taken over all distinct $\rho$ of the form $\lambda+\mu$, where $\lambda, \mu$ are $t$ th roots of unity. For example,

$$
\begin{aligned}
& P_{2}(x)=\frac{1}{2} x(x+2), \\
& P_{3}(x)=\frac{1}{12}\left(x^{3}+1\right)\left(x^{2}+2 x+4\right), \\
& P_{4}(x)=\frac{1}{80} x(x+2)\left(x^{2}+4\right)\left(x^{4}+4\right) .
\end{aligned}
$$

4. Does $P_{t}(x)$ characterize $G_{t}$ ? In view of the investigations of comparable questions for undirected graphs, it is natural to ask: if $H$ is a graph with $t^{2}$ vertices, and $P_{t}(x)$ is the polynomial of $H$, is $H \cong G_{t}$ ? We know of no instance in which $H \nsubseteq G_{t}$, but have only been able to prove $H \cong G_{t}$ if $t$ is a prime or if $t=4$. Before specializing to those cases, however, we begin with a few lemmas. We assume $H$ has $t^{2}$ vertices and belongs to $P_{t}(x)$, and $A$ is the adjacency matrix of $H$.

LEMMA 1. $H$ is strongly connected and strongly regular of valence 2.

Proof. That $H$ is strongly regular and strongly connected follows from the fact that $H$ has a polynomial. By Theorem 1 (iii) the valence of $H$ and the valence of $G_{t}$ both equal the largest real root of $P(x)=n$ and so the valence of $H=$ the valence of $G_{t}=2$.

Lemma 2. The vertices of $H$ can be partitioned into $t$ sets $T_{i}\left(i \in Z_{t}\right.$, 
the ring of residue classses $\bmod t)$, such that every edge in $H$ goes from $a$ vertex in $T_{i}$ to a vertex in $T_{i+1}$.

Proof. From the proof of Theorem 1, we know that 2 is an eigenvalue of $A$ of multiplicity one, and every eigenvalue is of absolute value at most 2 . Because $2 \lambda$ is also an eigenvalue of $A$ for $\lambda$ any $t$ th root of unity, it follows [2] that $A$ can be conceived as having the appearance

$$
\left(\begin{array}{llll}
0 & A_{0} & & \\
& 0 & A_{1} & 0 \\
& & 0 & \\
& & & A_{t-2} \\
A_{t-1} & & & 0]
\end{array}\right),
$$

where each diagonal block of 0 's is square. But each $A_{i}$ must also be square, since the numbers of 1 's in $A_{i}$ is twice the number of rows of $A_{i}$ and also twice the number of columns. Thus $A_{i}$ is of order $t$, which implies the lemma.

LEMma 3. Let $t>2$, let $\omega$ be a primitive th root of unity and let $\lambda$ be any $t$ th root of unity. Then for any $r, s$ with $(s, t)=1,1+\omega^{r}$ and $\lambda\left(1+\omega^{r s}\right)$ have the same multiplicities as eigenvalues of $A$.

Proof. Let $x$ be an eigenvector of $A$ corresponding to the eigenvalue $\alpha$, and let $x=\left(x_{0}, \cdots, x_{t-1}\right)$ denote the partitioning of the coordinates of $x$ corresponding to (4.1). We have $A_{i} x_{i+1}=\alpha x_{i}$. Thus $A_{i}\left(\lambda^{i+1} x_{i+1}\right)=\alpha \lambda\left(\lambda^{i} x_{i}\right)$. Thus $\left(x_{0}, \lambda x_{1}, \cdots, \lambda^{t-1} x_{t-1}\right)$ is an eigenvector of $A$ corresponding to the eigenvalue $\lambda \alpha$. Since the minimal polynomial of $A$ has no repeated factors, the multiplicity of an eigenvalue is just the dimension of the corresponding space of eigenvectors and so the multiplicities of $\alpha$ and $\lambda \alpha$ are the same. Finally the multiplicities of $1+\omega^{r}$ and $1+\omega^{\text {rs }}$ are the same, since these are algebraic conjugates and the characteristic polynomial of $A$ is rational. This concludes the proof of the lemma.

Note in particular that $2 \lambda$ is a simple eigenvalue of $A$.

LEMma 4. Let $A$ be of the form (4.1) and of rank $r$. Then for $0 \leqq i$ $\leqq t-1, j \geqq 0$, the rank of $A_{i} A_{i+1} A_{i+2} \cdots A_{i+j}$ is $r / t$, where addition of suffixes is taken $\bmod t$.

Proof. Let $m_{i}$ be the rank of $A_{i}$. Then $\sum_{i} m_{i}=$ the rank of $A=r$. Since the minimal polynomial of $A$ has no repeated factors, $A=S^{-1} D S$ for some nonsingular $S$ and diagonal $D$. Hence, the rank of $A^{t}$ is 
also $r$. Now $A^{t}$ consists of diagonal blocks $A_{0} A_{1} \cdots A_{t-1}, A_{1} A_{2} \cdots$ $A_{t-1} A_{0}, \cdots, A_{t-1} A_{0} \cdots A_{t-2}$. The rank of each block is at most $m=\min _{i} m_{i}$; hence, $\sum_{i} m_{i}=r=t m$ and so $m_{i}=r / t$ for all $i$. This proves the lemma for $j=0$. The result for $j>0$ follows from a similar consideration of $A^{i}$.

\section{Lemma 5. Let $t>2$. If $A$ is normal, then $H \cong G_{t}$.}

Proof. Let $K_{i}$ be the undirected bipartite graph whose vertices are the vertices of $T_{i}$ and $T_{i+1}$, as defined in Lemma 2, and which has an undirected edge joining $x \in T_{i}$ and $y \in T_{i+1}$ if and only if an edge of $H$ joins $x$ to $y$.

We first show that $K_{i}$ is a cycle of length $2 t$. Since every vertex of $K_{i}$ is of valence $2, K_{i}$ is the union of $p_{i}$ cycles for some $p_{i} \geqq 1$. The matrix $A A^{\mathrm{T}}$ has 4 as an eigenvalue with multiplicity $\sum_{i=1}^{t} p_{i}$. But since $A$ is normal, and $2 \lambda$ (for $\lambda$ any $t$ th root of unity) is a simple eigenvalue of $A, A A^{\mathrm{T}}$ has 4 as an eigenvalue with multiplicity $t$. Hence $p_{i}=1, i=0, \cdots, t-1$, which was to be proven.

Next, we show that trace $A^{t}=2 t^{2}$. Since each $K_{i}$ is a complete cycle the eigenvalues of $A A^{\mathrm{T}}$ are the union of the eigenvalues of $t$ matrices of order $t$ of the form $2 I+P_{i}+P_{i}^{\mathrm{T}}(i=0, \cdots, t-1)$, where each $P_{i}$ is a permutation matrix that represents a single cycle on $t$ letters. Therefore, $A A^{\mathrm{T}}$ has: 4 as an eigenvalue with multiplicity $t ; 2+\lambda+\bar{\lambda}$ as an eigenvalue with multiplicity $2 t$, for $\lambda=\exp (2 \Pi i k / t), k=1, \cdots$, $[(t-1) / 2]$; and if $t$ is even, 0 as an eigenvalue with multiplicity $t$.

Since $A$ is normal, these are the squares of the absolute values of the eigenvalues of $A$. Therefore, the number of eigenvalues of $A$ of a given absolute value (other than 2 or 0 ) is the same for each absolute value. We also know from Lemma 3 that all eigenvalues of the same absolute value occur equally often. It follows that $A$ has the same eigenvalues as the adjacency matrix for $G_{t}$. But the trace of the $t$ th power of that matrix is $2 t^{2}$, so trace $A^{t}=2 t^{2}$.

Since $K_{0}$ is a cycle of length $2 t$, we may label the vertices of $T_{0}$ and $T_{1}$ as $(i,-i)$ and $(i+1,-i)$, respectively $\left(i \in Z_{t}\right)$, in such a way that the edges from $(i,-i)$ go to $(i+1,-i)$ and $(i, 1-i)$. Since $A$ is normal and there is just one vertex, namely $(i,-i)$, which is the initial vertex of edges to both $(i+1,-i)$ and $(i, 1-i)$, it follows that there is just one vertex, which we label $(i+1,1-i)$, which is the terminal vertex of edges from both $(i+1,-i)$ and $(i, 1-i)$. We now have the vertices of $T_{1}$ and $T_{2}$ labelled in such a way that the edges from $(i+1,-i)$ go to $(i+2,-i)$ and $(i+1,1-i)$. We may continue labelling in this fashion the vertices of $T_{3}, T_{4}, \cdots, T_{t-1}$. Let $p_{i j}$ be the number of paths of length $t-1$ from $(i,-i)$ to $(j, t-1-j)$. 
Then $p_{i j}$ is $\left(\begin{array}{c}t-1 \\ m\end{array}\right)$ where $m$ is the least positive residue $(\bmod t)$ of $j-i$, for the normality of $A$ implies that the count of paths mimics the Pascal triangle. If $\left(\alpha_{i}, t-1-\alpha_{i}\right)$ and $\left(\beta_{i}, t-1-\beta_{i}\right)$ are the vertices of $T_{t-1}$ which are initial vertices of edges going to $(i,-i)$, then the number of paths of length $t$ from $(i,-i)$ to itself is $p_{i, \alpha_{i}}+p_{i, \beta_{i}}$. By hypothesis, trace $A^{t}=2 t^{2}$. Since the diagonal blocks of $A^{t}$ are cyclic permutations of the factors $A_{1}, A_{2}, \cdots, A_{t}$, each block has the same trace, $2 t$. Hence,

$$
\sum_{i=0}^{t-1}\left(p_{i, \alpha_{i}}+p_{i, \beta_{i}}\right)=2 t
$$

Since each $p_{i, \alpha_{i}}$ and $p_{i, \beta_{i}}$ is at least 1 , it follows that $p_{i, \alpha_{i}}$ and $p_{i, \beta_{i}}$ are exactly 1 and that $\alpha_{i}, \beta_{i}$ are just $i$ and $i-1$. We now have that the edges from $(i, t-1-i)$ go to $(i+1,-i-1)$ and $(i,-i)$ and have completed an explicit isomorphism between $G_{t}$ and $H$.

THEOREM 2. If $t=2,4$ or an odd prime, and $H$ is a graph with $t^{2}$ vertices that belongs to $P_{t}(x)$, then $H \cong G_{t}$.

Proof. We shall continue to use the notations of the lemmas.

If $t=2$ the classes $T_{i}$ of Lemma 2 each have 2 elements; hence the only possible distribution of edges is that of $G_{2}$.

If $t=4$ then the eigenvalues of $A$ are $\pm 2, \pm 2 i, \pm 1 \pm i$ and 0 . By Lemma 3 the eigenvalues $\pm 2, \pm 2 i$ are simple and the eigenvalues $\pm 1 \pm i$ have the same multiplicity, $m$ say. Since $A$ is of order 16 the multiplicity of 0 is $12-4 m$; hence $m=1$ or 2 . Suppose first that $m=1$, i.e., the multiplicity of 0 is 8 . Now, by Lemma 4 , each $A_{i}$ is of rank 2 and must therefore be of the form $P_{i} B Q_{i}$, where $P_{i}, Q_{i}$ are permutation matrices and

$$
B=\left(\begin{array}{llll}
1 & 1 & 0 & 0 \\
1 & 1 & 0 & 0 \\
0 & 0 & 1 & 1 \\
0 & 0 & 1 & 1
\end{array}\right) .
$$

Let $J_{4}$ be the $4 \times 4$ matrix of all 1 's. It may be readily verified that for a permutation matrix $R, B R B$ is one of $2 B, 2 J_{4}-2 B$ or $J_{4}$, and that $J_{4} R B$ is $2 J_{4}$. Hence

$$
\begin{aligned}
A_{1} A_{2} A_{3} A_{4} & =P_{1} B Q_{1} P_{2} B Q_{2} P_{3} B Q_{3} P_{4} B Q_{4} \\
& =8 P_{1} B Q_{4} \text { or } 8 P_{1}\left(J_{4}-B\right) Q_{4} \text { or } 4 P_{1} J_{4} Q_{4}=4 J_{4} .
\end{aligned}
$$

The third possibility cannot occur since, by Lemma $4, A_{1} A_{2} A_{3} A_{4}$ is of rank 2. 
Now $A^{4}$ is of the form

$$
\left(\begin{array}{cccc}
A_{1} A_{2} A_{3} A_{4} & 0 & 0 & 0 \\
0 & A_{2} A_{3} A_{4} A_{1} & 0 & 0 \\
0 & 0 & A_{3} A_{4} A_{1} A_{2} & 0 \\
0 & 0 & 0 & A_{4} A_{1} A_{2} A_{3}
\end{array}\right)
$$

and, as in the proof of Lemma 5, each of the diagonal blocks has the same eigenvalues and hence the same trace. From (4.2), the elements of $A_{1} A_{2} A_{3} A_{4}$ are divisible by 8 ; similarly for $A_{2} A_{3} A_{4} A_{1}, A_{3} A_{4} A_{1} A_{2}$ and $A_{4} A_{1} A_{2} A_{3}$. It follows therefore that the trace of $A^{4}$ is a multiple of 32 . On the other hand, the trace of $A^{4}=\sum \lambda^{4}$, the sum being over the eigenvalues $\lambda$ of $A$. On the assumption that $m=1$ these eigenvalues are, $2,2 i,-2,-2 i, 1+i, 1-i,-1+i,-1-i$, and 0 with multiplicity 8. A direct computation shows that $\operatorname{tr}\left(A^{4}\right)=48$. This contradicts the conclusion that $32 \mid \operatorname{tr}\left(A^{4}\right)$ and thus demonstrates the impossibility of the case $m=1$.

In the remaining case for $t=4$ the multiplicities of the eigenvalues are the same as those of the adjacency matrix of $G_{t}$. Hence the sum of the squares of the moduli of the eigenvalues of $A$ is $2 t^{2}$, which is the same as the sum of the squares of the elements of $A$. Therefore $A$ is normal. By Lemma $5, H \cong G_{t}$.

Finally, if $t$ is an odd prime, the eigenvalues of $A$ are just $2 \omega^{r}$ and $\omega^{r}+\omega^{s}$ for $0 \leqq r<s<t$, and $\omega$ a primitive $t$ th root of unity. We note that these numbers are all distinct. Now, by Lemma $3,2 \omega^{r}$ is a simple eigenvalue, and the eigenvalues $\omega^{r}+\omega^{s}$ all have the same multiplicity. This multiplicity must be 2 in order to account for all $t^{2}$ eigenvalues of $A$. We now have, as in the second case for $t=4$, that $A$ is normal, and $H \cong G_{t}$.

\section{REFERENCES}

1. A. J. Hoffman, On the polynomial of a graph, Amer. Math. Monthly 70 (1963), 30-36.

2. O. Perron, Zur Theorie der Matrizen, Math. Ann. 64 (1907), 248-263.

International Business Machines Corporation, Research Center, YorkTOWN Heights, New York 